\title{
Systematische Analyse von Medikationsfehlern mit Insulin
}

\author{
Olga Franka, Larissa Gehrigb, David Schwappach ${ }^{c}$ \\ ${ }^{a}$ Dr., Leitung Projekte, Produkte und Dienstleistungen Stiftung Patientensicherheit Schweiz; ${ }^{b}$ cand. MscN; ${ }^{c}$ Prof. Dr., MPH, Wissenschaftlicher Leiter \\ Stiftung Patientensicherheit Schweiz
}

\section{Zusammenfassung}

Medikationsfehler treten über den gesamten Medikationsprozess auf und können von folgenlosen Ereignissen bis zu schweren Schäden für den Patienten führen. Insulin gilt als Hochrisikomedikament. Deshalb ist im Umgang mit Insulin besondere Sorgfalt erforderlich. Die Stiftung Patientensicherheit Schweiz hat eine systematische Analyse der Fehlermeldungen in der CIRRNET-Datenbank durchgeführt, Medikationsfehler im Umgang mit Insulin analysiert und spezifische Problemfelder identifiziert. Medikationsfehler im Umgang mit Insulin treten in allen Schritten des Medikationsprozesses auf. Am häufigsten werden Fehlermeldungen bei der Verabreichung von Insulin beschrieben. Die dazugehörigen Problemfelder sind hauptsächlich Dosierungsfehler, Auslassungsfehler und Medikamentenverwechslungen. Da Insulin abhängig von Blutzuckerwerten und anderen Faktoren zeitnah verabreicht und die Dosierung regelmässig angepasst werden muss, stellt das Management von Diabetespatienten im Spital eine besondere Herausforderung dar.

\section{Hintergrund}

Diabetespatienten gehören zum klinischen Alltag eines jeden Spitals. Die Behandlung scheint besonders bei Patienten, die nicht primär wegen ihrer Diabeteserkrankung hospitalisiert werden, routiniert und verführt zu einem trügerischen Gefühl von Sicherheit. Besonders multimorbide Patienten können dabei Gefahr laufen, dass Nebendiagnosen eine weniger starke Beachtung bei der medizinischen Behandlung erfahren als die aktuell klinisch relevante Hauptdiagnose. Passieren Fehler, wie z.B. die Unterlassung einer regelmässigen Blutzucker-Kontrolle bei einem Diabetespatienten vor einer geplanten Intervention oder die Auslassung einer Insulinverabreichung vor der Nahrungszufuhr, besteht das Risiko von Blutzuckerentgleisungen mit potentiell gravierenden Folgeschäden für den Patienten.

Insulin zählt zu den Hochrisikomedikamenten [1], besitzt eine geringe therapeutische Breite und setzt genaue Dosisanpassungen mit vorsichtiger Verabreichung und regelmässige Überwachungen voraus. Aus- serdem sind die im Handel verfügbaren unterschiedlichen Darreichungsformen (z.B. Insulin-Spritzen, -Pens, -Pumpen usw.) und verschiedenen Insulin-Typen in verschiedenen Stärken und Formen eine grosse Herausforderung im korrekten Umgang mit Insulin.

Die Stiftung Patientensicherheit Schweiz hat aufgrund der hohen Relevanz der Medikationsfehler mit Insulin entschieden, CIRRNET-Fehlermeldungen (CIRRNET = Critical Incident Reporting \& Reacting NETwork) mit einer beschriebenen Insulinproblematik systematisch zu analysieren. Nachfolgend werden die Fehlermeldungen mit einer Insulinproblematik entlang des Medikationsprozesses (Medikationsprozess: 1. Verschreibung/Verordnung, 2. Dokumentation, 3. Ausgabe/Vorbereitung, 4. Verabreichung, 5. Monitoring) [2] dargestellt und aufgezeigt, welche spezifischen Problemfelder während der einzelnen Schritte im Medikationsprozess beschrieben werden und besonders häufig vorkommen.

\section{Methode}

CIRRNET ist ein Netzwerk lokaler Fehlermeldesysteme in der Schweiz, das seit 2006 von der Stiftung für Patientensicherheit betrieben wird. Die CIRRNET-Datenbank wurde im April 2015 systematisch mit definierten Stichworten auf Fehlermeldungen mit Insulin durchsucht. Insgesamt wurden 267 Fehlermeldungen gefunden, von denen nach erster Durchsicht 195 als relevante Fälle eingestuft wurden. Die ausgeschlossenen Fehlermeldungen hatten mit der Problematik Insulin im eigentlichen Sinn nichts zu tun, waren doppelt oder erwähnten die gesuchten Stichworte nur beispielhaft. Zwei unabhängig voneinander arbeitende Reviewer kategorisierten alle eingeschlossenen Fehlermeldungen nach den Schritten des Medikationsprozesses und identifizierten beschriebene Problemfelder. Aufgrund der nur sehr begrenzt zur Verfügung stehenden Informationen aus den Fehlermeldungen zeigte sich, dass sowohl die Kategorisierung nach dem Medikationsprozess als auch die Definition des beschriebenen Problemfeldes nicht immer eindeutig ausfallen kann. Die Reviewer entschieden sich deshalb für einen Probelauf einer Kategorisierung mit einer Zufallsstichprobe, 
darauf folgender Diskussion für ein gemeinsames Verständnis und anschliessender, unabhängig voneinander durchgeführter Kategorisierung und Analyse aller eingeschlossenen Fehlermeldungen.

\section{Ergebnisse}

Die Analyse der eingeschlossenen CIRRNET-Meldungen durch zwei Reviewer ergab bei der Zuordnung zu den Schritten des Medikationsprozesses eine Übereinstimmung von $80 \%(n=212)$. Nicht übereinstimmend zugeordnete Fehlermeldungen wurden im Konsens durch beide Reviewer nachträglich gemeinsam zugeordnet. Die Anzahl CIRRNET-Meldungen je Prozessschritt und die identifizierten Problemfelder sind in der Tabelle 1 detailliert aufgeführt.
Beim ersten Prozessschritt Verschreibung/Verordnung (13,3\%) konnten sieben Problemfelder aus 26 Meldungen identifiziert werden. Am häufigsten werden vergessene Verordnungen von Insulin (3,6\%) beschrieben. Unklare Verordnungen $(3,1 \%)$ sind ein weiteres Problemfeld, das sich z.B. durch Widersprüchlichkeiten bei den Angaben von Insulinlaufraten zeigt. Im Zusammenhang mit Nachspritzschemata werden insbesondere mehrfache Verordnungen $(2,6 \%)$ aufgeführt, welche unterschiedlich ausgefüllt und/oder mehrfach für ein und denselben Patienten ausgestellt sind. Zudem wird über unleserliche und/oder unvollständige Verordnungen berichtet und nicht zuletzt über die fehlende Kommunikation von Neuverordnungen oder die fehlende Kommunikation von geänderten Verordnungen durch den Arzt an die Pflegefachpersonen berichtet.

\begin{tabular}{|c|c|c|c|c|c|}
\hline Prozessschritt & Problemfeld & $\begin{array}{l}\text { Anzahl } \\
\text { Fälle }\end{array}$ & Total & $\begin{array}{l}\text { Anteil } \\
\text { Fälle }\end{array}$ & Total \\
\hline \multirow[t]{7}{*}{ 1. Verschreibung/Verordnung } & fehlende Verordnung & 7 & 26 & $3,59 \%$ & $13,3 \%$ \\
\hline & unklare Verordnung & 6 & & $3,08 \%$ & \\
\hline & unleserliche Verordnung & 1 & & $0,51 \%$ & \\
\hline & unvollständige Verordnung & 1 & & $0,51 \%$ & \\
\hline & falsche Verordnung & 5 & & $2,56 \%$ & \\
\hline & mehrfache Verordnung & 5 & & $2,56 \%$ & \\
\hline & fehlende Kommunikation von Neuverordnungen und Änderungen & 1 & & $0,51 \%$ & \\
\hline \multirow[t]{7}{*}{ 2. Dokumentation } & fehlende Dokumentation & 5 & 19 & $2,56 \%$ & $9,7 \%$ \\
\hline & unklare Dokumentation & 2 & & $1,03 \%$ & \\
\hline & unleserliche Dokumentation & 1 & & $0,51 \%$ & \\
\hline & unvollständige Dokumentation & 3 & & $1,54 \%$ & \\
\hline & falsche Dokumentation & 2 & & $1,03 \%$ & \\
\hline & Übertragungsfehler & 5 & & $2,56 \%$ & \\
\hline & unsicherer Umgang mit elektronischem Dokumentationssystem & 1 & & $0,51 \%$ & \\
\hline \multirow[t]{4}{*}{ 3. Ausgabe/Vorbereitung } & falsche Zubereitung & 1 & 7 & $0,51 \%$ & $3,6 \%$ \\
\hline & falsches Medikament & 3 & & $1,54 \%$ & \\
\hline & fehlende Beschriftung & 1 & & $0,51 \%$ & \\
\hline & Berechnungsfehler & 2 & & $1,03 \%$ & \\
\hline \multirow[t]{10}{*}{ 4. Verabreichung } & Dosierungsfehler & 36 & 132 & $18,46 \%$ & $67,7 \%$ \\
\hline & Medikamentenverwechslung & 27 & & $13,85 \%$ & \\
\hline & Auslassungsfehler & 36 & & $18,46 \%$ & \\
\hline & Nichteinhalten von Verordnungen & 8 & & $4,10 \%$ & \\
\hline & Doppelte Verabreichung & 1 & & $0,51 \%$ & \\
\hline & falscher Verabreichungszeitpunkt & 4 & & $2,05 \%$ & \\
\hline & Patientenverwechslung & 14 & & $7,18 \%$ & \\
\hline & Fehler bei Selbstmedikation & 4 & & $2,05 \%$ & \\
\hline & Inkompatibilität von Arzneimitteln & 1 & & $0,51 \%$ & \\
\hline & falsche Applikationsart (s.c./i.v.) & 1 & & $0,51 \%$ & \\
\hline \multirow[t]{4}{*}{ 5. Monitoring } & fehlende adäquate Anpassung bei sich klinisch verändernder Patientensituation & 3 & 11 & $1,54 \%$ & $5,6 \%$ \\
\hline & ausgelassenes/verzögertes Eintrittsmanagement von Diabetespatienten & 5 & & $2,56 \%$ & \\
\hline & Verwendung falsches Equipment & 2 & & $1,03 \%$ & \\
\hline & $\begin{array}{l}\text { mangelnde/fehlende Kommunikation von Befunden, Verordnungen an } \\
\text { Schnittstellen }\end{array}$ & 1 & & $0,51 \%$ & \\
\hline Gesamt & & 195 & 195 & $100 \%$ & $100 \%$ \\
\hline
\end{tabular}


Auch bei der Dokumentation beschreiben die CIRRNET-Meldungen unterschiedliche Fehler (9,7\%). So werden z.B. Übertragungsfehler $(2,6 \%)$ aufgeführt, die bei der Verwendung von nicht elektronischen Dokumentationssystemen immer wieder vorkommen. Vor einer fehlenden Dokumentation (2,6\%) schützen aber auch keine elektronische Dokumentationssysteme, wie die CIRRNET-Meldungen zeigen.

Am häufigsten beschreiben die analysierten CIRRNET-Meldungen Fehler im Prozessschritt der Verabreichung von Insulin (67,7\%). Dosierungsfehler in Form von Über- oder Unterdosierungen (18,5\%) und Auslassungsfehler (18,5\%) sind dabei am häufigsten anzutreffen. Die beschriebenen Dosierungsfehler sind sehr unterschiedlicher Art. So wird z.B. beschrieben, dass beim Insulin "Novomix 30" die Ziffer im Medikamentennamen bei handschriftlicher Dokumentation fälschlicherweise als Angabe für die zu verabreichende Dosis gelesen und verabreicht wurde. Die Auslassungsfehler werden unter anderem als Vergessen einer Verabreichung im Rahmen einer Delegation von Tätigkeiten oder als Vorkommen bei erhöhtem Arbeitsaufwand beschrieben. Ausserdem wird über Medikamentenverwechslungen (13,9\%) von Langzeit-/Kurzzeitinsulinen berichtet, aber auch über Verwechslungen von Insulin mit ähnlich klingendem Namen wie z.B. «Levemir» und «Lantus». Es werden jedoch nicht nur das Insulin verwechselt sondern auch die Patienten. So beschreiben 14 Fehlermeldungen $(7,2 \%)$ eine Patientenverwechslung, bei der z.B. der Nachbarpatient das Insulin erhält oder gar ein Patient ohne eine Indikation für die Insulinverabreichung (kein Diabetiker). Des Weiteren wird über den falschen Zeitpunkt (2,1\%) der Insulinverabreichung berichtet oder auch über vielschichtige Probleme mit Patienten, welche selbständig ihr Insulin während des Spitalaufenthalts spritzen (2,1\%). Die Analyse der CIRRNET-Meldungen liefert zudem interessante Ergebnisse in Bezug auf das Eintrittsmanagement von Diabetespatienten. So werden z.B. bei Patienten, die mit der Nebendiagnose Diabetes mellitus eintreten, vergessene oder verzögerte ärztliche Verordnungen für die Diabetesbehandlung beschrieben (2,6\%). Oder die verzögerte Reaktion auf eine sich akut verändernde klinische Situation des Patienten durch z.B. eine Hypo- oder Hyperglykämie (1,5\%). Die genaue und zeitnahe Dosisanpassung bei sich klinisch verändernden Blutzuckerwerten und die dazugehörige engmaschige und regelmässige Blutzuckerüberwachung gehören zu den grössten Herausforderungen im Klinikalltag und zeigen sich besonders bei sehr grosser Arbeitsbelastung des Klinikpersonals.

\section{Schlussfolgerung}

Das Ziel dieser Analyse war, Fehlermeldungen der CIRRNET-Datenbank mit einer Insulinproblematik systematisch auszuwerten und spezifische Problemfelder im Umgang mit Insulin zu identifizieren. Die Ergebnisse reihen sich neben die Analyseergebnisse anderer internationaler Institutionen ein. So ergab die Auswertung des National Reporting and Learning System [3] in Grossbritannien, dass die Hauptprobleme im Umgang mit Insulin falsche Dosierungen, vergessene Verabreichungen oder Medikamentenverwechslungen waren - Problemfelder, welche dem Prozessschritt der Verabreichung zugeordnet werden können.

Die Ergebnisse sind vor dem Hintergrund zu interpretieren, dass nicht bekannt ist, von welcher Berufsgruppe die analysierten CIRRNET-Meldungen berichtet wurden und von einem entsprechenden Bias auszugehen ist. Sie liefern jedoch hervorragende Informationen für das Qualitäts- und Risk-Management und für eine verbesserte Patientensicherheit beim Umgang mit Insulin. In diesem Sinne bieten besonders auch die Einzelfälle mit ungewöhnlichen Vorkommnissen die Möglichkeit, seltene, aber mit grossem Schadenspotential einhergehende Fälle zu erkennen und Verbesserungsmassnahmen einzuleiten. So zeigte z.B. die Meldung mit der irreführenden Produktebezeichnung «Novomix 30» ein Problemfeld auf, das möglicherweise sonst nicht erkannt worden wäre. Die Auslassungsfehler hingegen sind Fachpersonen bekannt und werden durch die Häufigkeit der Fehlermeldungen zu diesem Problemfeld bestätigt. Besonders häufig werden die Auslassungsfehler im Zusammenhang mit einer hohen Arbeitsbelastung beschrieben. Dazu kommt, dass die Verabreichung von Insulin entsprechend der aktuellen Blutzuckerwerte erfolgen muss und die voneinander abhängige Koordination der Blutzuckerwerte und der Insulindosis zeitnahe zu erfolgen hat und bei erhöhtem Arbeitsaufwand oftmals einfach vergessen wird.

Ein Augenmerk sollte auch auf das Eintrittsmanagement von Diabetespatienten gelegt werden, die mit einer anderen Hauptdiagnose hospitalisiert werden und die Nebendiagnose des Diabetes mellitus, die eine zeitnahe Verordnung und Therapie voraussetzt, zu wenig Aufmerksamkeit erhält.

Der Umgang mit Insulin und das Insulinmanagement von Diabetespatienten sind verbesserungswürdig. Die Analyseergebnisse der CIRRNET-Meldungen liefern Hinweise auf bestehende Problemfelder. Jede Gesundheitseinrichtung sollte deshalb den eigenen Umgang mit Insulin individuell auf Schwachstellen überprüfen und somit zu einer erhöhten Medikationssicherheit und Patientensicherheit beitragen. 